\title{
Indifferent electromagnetic modes: bound states and topology
}

\author{
S. A. R. Horsley* \\ Department of Physics and Astronomy, Stocker Road, University of Exeter, Exeter EX4 4QL
}

\begin{abstract}
At zero energy the difference in the number of spin up and spin down modes of the Dirac equation is determined by the topology of both space, and the gauge field in which the system sits. Writing Maxwell's equations in a Dirac-like form, we identify cases where a combination of material parameters plays the role of 'energy'. At zero 'energy' we thus find electromagnetic modes that are indifferent to local changes in the material parameters, with a dispersion relation depending only on the asymptotic values of the material parameters at infinity. We give several examples, and show that this theory has implications for non-Hermitian media, where it can be used to construct permittivity profiles that are either reflectionless, or act as coherent perfect absorbers, or lasers.
\end{abstract}

Most physical theories are concerned with the local behaviour of physical variables, such as the electromagnetic or graviational field. By contrast, topology is the mathematical subject concerned with finding invariant global properties of abstract objects. Nevertheless there are some fascinating examples where topology has been applied to physics: the vacuum energy of a scalar field is sensitive to the topology of space [1], and topology characterizes Skyrmions in magnetic systems [2]; defects in liquid crystals [3]; vacuum states in non-Abelian gauge theories [4]; the theory of general relativity in $2+1$ dimensions [5]; and the theory of topological insulators in condensed matter physics [6].

This work applies topological methods to electromagnetic materials, predicting mode characteristics that are independent of the detailed inhomogeneity of the material. The results are rather different from theories such as transformation optics [7-9], where the function of a device depends on an accurate implementation of the material tensors across space. Topological results are by definition insensitive to the local details of the material, and have already been shown to govern the number of interface states between adjoining materials [10-13]. The theory has been developed for both continuous and periodic media [14-16], and has been connected to the properties of the Dirac equation [17, 18], which also plays a role in this work. Because these trapped interface states can be predicted using topological methods, they are rather robust to the details of the interface, and have been experimentally observed to propagate past extreme obstacles without backscattering [11].

Here we find an interesting set of electromagnetic modes whose existence only depends on the behaviour of the material parameters at infinity (or rather, large distances from the inhomogeneity). For example, we find a family of media with modes that have a dispersion relation that is invariant to local changes to the material. To find these solutions we make use of an analogy between Maxwell's equations in inhomogeneous media and the Dirac equation $[17,19]$. To understand this analogy consider the Dirac equation in two dimensions, for a particle

\footnotetext{
*s.horsley@exeter.ac.uk
}

of mass $m$ and energy $\mathcal{E}$ in a gauge field $\boldsymbol{A}=A_{x} \hat{\boldsymbol{x}}+A_{y} \hat{\boldsymbol{y}}$

$$
\left(\begin{array}{cc}
0 & \mathcal{D} \\
\mathcal{D}^{\dagger} & 0
\end{array}\right)\left(\begin{array}{l}
\psi_{+} \\
\psi_{-}
\end{array}\right)=\left(\begin{array}{cc}
\mathcal{E}-m & 0 \\
0 & \mathcal{E}+m
\end{array}\right)\left(\begin{array}{l}
\psi_{+} \\
\psi_{-}
\end{array}\right)
$$

where $\mathcal{D}=-2 \mathrm{i} \partial / \partial z-A_{+}, \psi_{+,-}$are the wavefunctions for the two spin components, $z=x+\mathrm{i} y$, and $A_{+}=A_{x}-\mathrm{i} A_{y}$. Besides being a limiting case of the relativistic description of electrons, this equation appears as an effective description in planar optics, notably in deformed honeycomb lattices [20,21] and gyrotropic media [17]. Rather unexpectedly, Maxwell's equations for fields of a fixed frequency $\omega$ can be written in a very similar form to (1) if the electric $\boldsymbol{E}$ and magnetic $\boldsymbol{H}$ fields are combined into a single six-vector [17],

$$
\left(\begin{array}{cc}
\mathbf{0} & \mathcal{D} \\
\mathcal{D}^{\dagger} & \mathbf{0}
\end{array}\right)\left(\begin{array}{c}
\boldsymbol{E} \\
\eta_{0} \boldsymbol{H}
\end{array}\right)=-k_{0}\left(\begin{array}{cc}
\boldsymbol{\epsilon} & \mathbf{0} \\
\mathbf{0} & \boldsymbol{\mu}
\end{array}\right)\left(\begin{array}{c}
\boldsymbol{E} \\
\eta_{0} \boldsymbol{H}
\end{array}\right)
$$

where $\eta_{0}=\sqrt{\mu_{0} / \epsilon_{0}}$ is the impedance of free space, $k_{0}=\omega / c$ is the free space wavenumber, and $\boldsymbol{\epsilon}, \boldsymbol{\mu}$ and $\boldsymbol{\xi}$ are respectively the permittivity, permeability and bianisotropy tensors for a lossless medium. In this case the differential operator is given by $\mathcal{D}=-\mathrm{i} \boldsymbol{\nabla} \times+k_{0} \boldsymbol{\xi}$. A comparison between equations (1) and (2) shows that, broadly speaking the electric and magnetic fields play the role of the two spin components in an effective Dirac equation: the bianisotropy plays the role of the gauge field, the 'energy' is given by the average of the permeability and permittivity $-k_{0}(\boldsymbol{\mu}+\boldsymbol{\epsilon}) / 2$, and the 'mass' by half their difference $k_{0}(\boldsymbol{\epsilon}-\boldsymbol{\mu}) / 2$. Although the vector nature of the wavefunction components make this analogy incomplete, for one dimensional variations we shall show it can be made exact.

Now for the role of topology: if both the energy $\mathcal{E}$ and mass $m$ are zero in the Dirac equation (1), the two spin components $\psi_{ \pm}$become decoupled, satisfying

$$
\begin{aligned}
\mathcal{D} \psi_{-} & =0 \\
\mathcal{D}^{\dagger} \psi_{+} & =0 .
\end{aligned}
$$

The difference in the number of solutions to $(3), N=$ $\operatorname{dim}[\operatorname{ker} \mathcal{D}]$ and the solutions to $(4), \bar{N}=\operatorname{dim}\left[\operatorname{ker} \mathcal{D}^{\dagger}\right]$ is governed by a rather deep and far-ranging result in topology known as the Atiyah-Singer index theorem [22, 23]. 
This theorem is an extreme generalization of the GaussBonnet theorem [23], and has been connected to the aforementioned work on interface states between periodic media $[24,25]$. In general it states that

$$
\begin{aligned}
\operatorname{index}[\mathcal{D}] & =N-\bar{N} \\
& =\int_{M} \hat{A}(M) \wedge \operatorname{ch}(V)
\end{aligned}
$$

where the integration is taken over the manifold of interest $M$, ' $\wedge$ ' is the exterior product, $\hat{A}(M)$ is the 'Ahat genus', depending on the curvature of the space, and $\operatorname{ch}(V)$ is the 'Chern character' depending on the curvature of the gauge field [26-28]. To put this theorem in physical terms, the difference in the number of solutions $N-\bar{N}$ cannot be altered though any continuous change of the system parameters. The implications of this index theorem are well known for the true Dirac equation, but do not seem to have been considered seriously in electromagnetism. Are electromagnetic modes also controlled by this theorem? This seems a natural question to ask, given the close similarity between (1) and (2). Restricting ourselves to planar media, we find that this theorem indeed dictates the existence of many electromagnetic modes, and that these turn out to be analogous to the so-called 'Jackiw-Rebbi modes' of the Dirac equation. This connects several existing results in the literature, from photonic topological insulators to non-Hermitian reflectionless media, as well as predicting new materials exhibiting modes with a dispersion relation that is insensitive to local variations of the material parameters.

\section{A. Jackiw-Rebbi modes}

Before considering the electromagnetic case, we review a one dimensional example of the Dirac equation where the allowed modes depend on the behaviour of the system at infinity, and show that this behaviour is connected to the index of the operator appearing in (5). The modes given in this section are the so-called JackiwRebbi modes of the Dirac equation [29]. In the field of toplogical insulators these modes are typically connected with the Chern number, computed as an integral over $\boldsymbol{k}$ space [6]. Here instead we show that more directly (and more generally) this mode can be connected with the index of a first order operator.

The two-dimensional Dirac equation (1) with zero magnetic field takes the following form

$$
\left[-\mathrm{i} \sigma_{x} \partial_{x}-\mathrm{i} \sigma_{y} \partial_{y}+m(x) \sigma_{z}\right]|\psi\rangle=\mathcal{E}|\psi\rangle
$$

where we allow the particle mass to depend on position, and $|\psi\rangle$ is the two component wavefunction. Assuming translational symmetry along $y\left(-\mathrm{i} \partial_{y} \rightarrow k_{y}\right)$, and writing the wave in terms of the eigenfunctions $a_{y, \pm}$ of $\sigma_{y}$ with eigenvalue \pm 1

$$
|\psi\rangle=\psi_{+} a_{y,+}+\psi_{-} a_{y,-}
$$
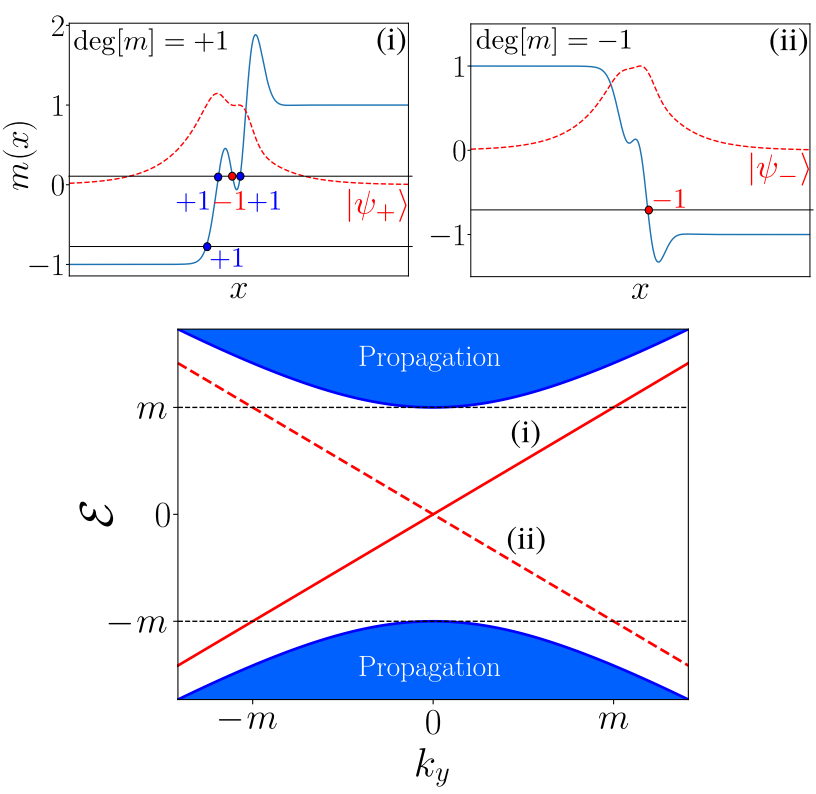

FIG. 1. Jackiw-Rebbi modes of the Dirac equation (6) with position dependent mass. Different arbitrarily chosen mass distributions are shown as solid blue curves in panels (i) and (ii), plus mode profiles $\psi_{ \pm}$(red dashed lines, computed from (9)). The mass profiles can be classified in terms of their topological degree (see Eq. (11)), the calculation of which is performed through summing intersection points along the thin horizontal lines as indicated in (i-ii). The degree is a topological invariant, independent of the choice of horizontal line (assuming the mass ultimately diverges at infinity, without changing sign). The lower plot sketches the dispersion relation for the Dirac equation (6), where for $\mathcal{E}>m$ (shaded blue) we have propagation in the region of space where $m \sim$ const.. The red lines crossing the 'mass gap' $\mathcal{E} \in[-m, m]$ indicate the dispersion of the two modes shown in the upper panels.

where $a_{y, \pm}=(1, \pm \mathrm{i})^{T}$, the Dirac equation (6) can be reduced to the form

$$
\left(\begin{array}{cc}
0 & \mathcal{D} \\
\mathcal{D}^{\dagger} & 0
\end{array}\right)\left(\begin{array}{l}
\psi_{+} \\
\psi_{-}
\end{array}\right)=\left(\begin{array}{cc}
\mathcal{E}-k_{y} & 0 \\
0 & \mathcal{E}+k_{y}
\end{array}\right)\left(\begin{array}{l}
\psi_{+} \\
\psi_{-}
\end{array}\right)
$$

where $\mathcal{D}=-\partial_{x}+m(x)$. From the above pair of equations (8) we see that the difference in the number $N$ of solutions to $\mathcal{D} \psi_{-}=0$ and the number $\bar{N}$ of solutions to $\mathcal{D}^{\dagger} \psi_{+}=0$ is equivalent to the difference in the number of modes with $\mathcal{E}=-k_{y}$ and those with $\mathcal{E}=k_{y}$. For positive energy within a gap, where propagation in the bulk is disallowed, this is the difference in the number of modes propagating down or up the $y$ axis.

As discussed above, the difference $N-\bar{N}$ is fixed by the behaviour of $m(x)$ at infinity. We do not need the sophisticated mathematics of (5) to see this: it is immediately evident from the solutions to (8),

$$
\psi_{ \pm}=\exp \left(\mp \int_{0}^{x} m\left(x^{\prime}\right) d x^{\prime}\right) \quad\left(\mathcal{E}= \pm k_{y}\right)
$$

The solutions (9) are the well-known Jackiw-Rebbi 
modes [29] that occur between regions where the mass has a different sign. If $m(x)$ has the same sign at $x=+\infty$ and $x=-\infty$ then neither of the states (9) is normalizable and $N=\bar{N}=0$. Conversely if $m(x)$ takes a different sign at these two limits then $N-\bar{N}$ equals \pm 1 , for the respective cases of negative and positive $m$ at $+\infty$. A compact way of writing this result is in terms of the mapping degree of $\operatorname{deg}[m(x)][30]$

$$
\operatorname{index}[\mathcal{D}]=N-\bar{N}-=-\operatorname{deg}[m(x)]= \pm 1,0,
$$

where the degree is defined as

$$
\operatorname{deg}[m(x)]=\sum_{x_{a} \in m^{-1}(a)} \operatorname{sign}\left[m^{\prime}\left(x_{a}\right)\right] .
$$

In this one dimensional case the degree is the topological invariant appearing on the right hand side of the index theorem (5). Examples are shown in panels (i) and (ii) of Fig. 1. As can be established from an examination of Fig. 1, strictly speaking the mass $m(x)$ should diverge at infinity for the degree $\operatorname{deg}[m]$ to be well defined [31]. In practice however, the modes we predict do not depend on this restriction.

It is also possible to write the invariant (11) in terms of a winding number, corresponding to the behaviour of the Dirac Hamiltonian in $\boldsymbol{k}$ space at spatial infinity [32]. We do not take this approach here. Firstly the degree is simpler, but more importantly the parameters in our Dirac-like equation (e.g. the prefactor of $\sigma_{y}$ ) cannot in general be identified with points in $\boldsymbol{k}$-space.

\section{B. Electromagnetic modes in stratified media}

In electromagnetic terms the above modes (9) are bound modes in stratified media where the dispersion relation connecting the frequency and wave-vector is insensitive to the specific spatial distribution of the material parameters. We now explain the problem of finding the modes of generic stratified electromagnetic materials (schematic shown in Fig. 2), illustrating why confined modes usually have a dispersion relation sensitive to the precise distribution of material parameters.

For generic stratified media inhomogeneous along $x$, Maxwell's equations (2) reduce to

$$
\mathrm{i} \Gamma_{1} \frac{d}{d x}\left(\begin{array}{c}
\boldsymbol{E} \\
\eta_{0} \boldsymbol{H}
\end{array}\right)=k_{0}\left(\begin{array}{cc}
\boldsymbol{\epsilon} & \overline{\boldsymbol{\xi}} \\
\overline{\boldsymbol{\xi}}^{\dagger} & \boldsymbol{\mu}
\end{array}\right)\left(\begin{array}{c}
\boldsymbol{E} \\
\eta_{0} \boldsymbol{H}
\end{array}\right)=k_{0} \chi\left(\begin{array}{c}
\boldsymbol{E} \\
\eta_{0} \boldsymbol{H}
\end{array}\right)
$$

where we have introduced a set of matrices $\Gamma_{j}$ to represent the curl operator

$$
\Gamma_{j}=\left(\begin{array}{cc}
\mathbf{0} & \boldsymbol{L}_{j} \\
-\boldsymbol{L}_{j} & \mathbf{0}
\end{array}\right)
$$

with the three $\boldsymbol{L}_{j}$ matrices given by

$$
\boldsymbol{L}_{1}=\left(\begin{array}{ccc}
0 & 0 & 0 \\
0 & 0 & -1 \\
0 & 1 & 0
\end{array}\right), \boldsymbol{L}_{2}=\left(\begin{array}{ccc}
0 & 0 & 1 \\
0 & 0 & 0 \\
-1 & 0 & 0
\end{array}\right), \boldsymbol{L}_{3}=\left(\begin{array}{ccc}
0 & -1 & 0 \\
1 & 0 & 0 \\
0 & 0 & 0
\end{array}\right)
$$

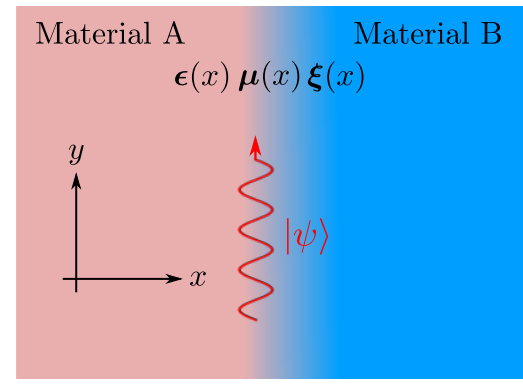

FIG. 2. Schematic of configuration. We consider an electromagnetic material that is inhomogeneous along $x$ (in this example tending to uniformity at infinity), and look for bound electromagnetic modes $|\psi\rangle$ (here confined to the interface). In general the bound modes have a dispersion relation that is sensitive to the specific spatial distribution of the material parameters (found from the general result (19)).

The propagation constants along $y$ and $z$ are $k_{y}$ and $k_{z}$ respectively, and we have combined the terms arizing from this propagation into a modified bianisotropy tensor $\overline{\boldsymbol{\xi}}$

$$
\overline{\boldsymbol{\xi}}=\boldsymbol{\xi}+\frac{k_{y}}{k_{0}} \boldsymbol{L}_{2}+\frac{k_{z}}{k_{0}} \boldsymbol{L}_{3}
$$

The problem with comparing equation (12) to the Dirac equation is that $\Gamma_{1}$ is not an element of a Clifford algebra. This is due to the transverse nature of the electromagnetic field, where any field vector with $\boldsymbol{E}$ and $\boldsymbol{H}$ pointing along $\hat{\boldsymbol{x}}$ is reduced to zero by $\Gamma_{1}$ (and hence the $x$ components of the fields do not appear on the left of (12)). For planar media we can sidestep this difficulty through solving (12) for the field components $E_{x}$ and $H_{x}$ normal to the interface, finding that

$$
\left(\begin{array}{c}
E_{x} \\
\eta_{0} H_{x}
\end{array}\right)=-\chi_{x x}^{-1} \chi_{x p}\left(\begin{array}{c}
\boldsymbol{E}_{\|} \\
\eta_{0} \boldsymbol{H}_{\|}
\end{array}\right)
$$

where $\chi_{x x}$ is the $2 \times 2$ matrix with elements $\epsilon_{x x}, \bar{\xi}_{x x}, \bar{\xi}_{\underline{\underline{x}} x}^{\star}$, and $\mu_{x x}$ and $\chi_{x p}$ is a $2 \times 4$ matrix with elements $\boldsymbol{\epsilon}_{\boldsymbol{x} \|}, \boldsymbol{\xi}_{x \|}$ etc., and a subscript $\|$ indicates components in the $y-z$ plane. Using the result (16) to eliminate these $\hat{\boldsymbol{x}}$ field components, Eq. (12) reduces to

$$
\mathrm{i} \gamma_{1} \frac{d}{d x}|\psi\rangle=k_{0} \gamma_{1} \gamma_{2}\left[\chi_{p p}-\chi_{p x} \chi_{x x}^{-1} \chi_{x p}\right]|\psi\rangle
$$

where $|\psi\rangle=\left(\boldsymbol{E}_{\|}, \eta_{0} \boldsymbol{H}_{\|}\right)^{\mathrm{T}}$, and the $4 \times 4 \gamma_{j}$ matrices are of the usual Dirac form

$$
\gamma_{0}=\left(\begin{array}{cc}
\mathbf{1}_{2} & 0 \\
0 & -\mathbf{1}_{2}
\end{array}\right), \gamma_{j}=\left(\begin{array}{cc}
0 & \sigma_{j} \\
-\sigma_{j} & 0
\end{array}\right), \gamma_{5}=\left(\begin{array}{cc}
0 & \mathbf{1}_{2} \\
\mathbf{1}_{2} & 0
\end{array}\right) .
$$

and now form a Clifford algebra. In a planar geometry in general we can thus reduce Maxwell's equations to a form (17) that is analogous to the four component Dirac equation, with the material parameters corresponding to generally rather complicated contributions to the Hamiltonian. One advantage of recasting Maxwell's equations 
into this Dirac-like form is that it is immediately evident that the solution to Eq. (17) can be written in terms of a path ordered exponential

$$
|\psi\rangle=\mathrm{P}\left[\mathrm{e}^{-\mathrm{i} k_{0} \int_{0}^{x} \gamma_{2}\left(\chi_{p p}-\chi_{p x} \chi_{x x}^{-1} \chi_{x p}\right) d x^{\prime}}\right]\left|\psi_{0}\right\rangle
$$

where $\left|\psi_{0}\right\rangle$ is the form of the in-plane electromagnetic field at $x=0$. Importantly this is the general solution to Maxwell's equations in a layered material.

The result given in Eq. (19) already resembles the Jackiw-Rebbi mode (9). However they are crucially not the same, and this reveals why the dispersion of confined electromagnetic modes is almost always sensitive to the precise distribution of the material parameters. Firstly, the exponent of (19) is not generally a Hermitian operator. This means that the exponent can be either real or complex valued. Secondly, the path ordering is necessary because the basis vectors of the matrix in the exponent (i.e. the polarization basis) will change with position, continually rotating $\left|\psi_{0}\right\rangle$ as we move along the $x$ axis. The combination of these two properties means that an arbitrary choice of $\left|\psi_{0}\right\rangle$ in Eq. (19) will most often either be propagating or divergent at infinity, rather than tending to zero as the mode (9) does.

To find the bound modes in a particular material profile one must carefully choose $k_{y}, k_{z}$, and $k_{0}$ such that the field amplitude vanishes asymptotically. This careful choice is of course equivalent to the dispersion relation of the mode, and due to the path ordering this choice must be made differently if the distribution of the material properties is changed. From this perspective, electromagnetic Jackiw-Rebbi modes are those special families of material parameters where the path ordering can be dropped from (19). Using our analogy with the Dirac equation, we shall now show examples of such confined modes, the existence of which can also be understood in terms of the topological invariant (11).

At this point it may be useful to give an analogy. Our calculation may sound rather exotic, but there is a very simple well-known example of the kind of mode we are looking for. Consider a parallel plate waveguide, where the plates cover the $y-z$ plane, are highly conducting, and separated by a distance $L$. For nearly all the modes, the dispersion relation depends on the plate separation as e.g. $k_{y}^{2}=k_{0}^{2}-(n \pi / L)^{2}$. This is true except for the $n=$ 0 mode, which always has the same dispersion relation $k_{y}^{2}=k_{0}^{2}$, and is insensitive to the plate separation. Here we are using the topological invariant (11) to find the analogous guided modes in graded material profiles.

\section{Examples of electromagnetic Jackiw-Rebbi modes}

To keep the discussion simple we do not work in terms of our general solution (19), but rather specialize to a particular case of the optical Dirac equation (17). Assuming zero propagation constant along $z$, and modes that are either $E_{z}$ or $H_{z}$ polarized waves (i.e. have either their electric, or magnetic fields pointing only along the $\hat{\boldsymbol{z}}$ axis), we are restricted to the following form of the material tensors

$$
\boldsymbol{\epsilon}=\left(\begin{array}{cc}
\boldsymbol{\epsilon}_{\|} & \mathbf{0} \\
\mathbf{0} & \epsilon_{z z}
\end{array}\right), \boldsymbol{\mu}=\left(\begin{array}{cc}
\boldsymbol{\mu}_{\|} & \mathbf{0} \\
\mathbf{0} & \mu_{z z}
\end{array}\right), \boldsymbol{\xi}=\left(\begin{array}{cc}
\mathbf{0} & \boldsymbol{v}^{T} \\
\boldsymbol{w} & 0
\end{array}\right) .
$$

where $\boldsymbol{v}$ and $\boldsymbol{w}$ are two element complex vectors, i.e. $\boldsymbol{v}=\left(v_{x}, v_{y}\right)$. With these assumptions the optical Dirac equation (17) reduces to a pair of uncoupled two component Dirac-like equations. For the $E_{z}$ polarization the equation is given by

$$
\left[-\mathrm{i} \sigma_{x}\left(k_{0}^{-1} \frac{d}{d x}+\mathrm{i} \alpha_{1}\right)-\alpha_{2} \sigma_{y}+m \sigma_{z}\right]|\psi\rangle=\mathcal{E}|\psi\rangle
$$

where $|\psi\rangle=\left(E_{z}, \eta_{0} H_{y}\right)^{\mathrm{T}}$, and the complex number $\alpha$ is given by

$$
\alpha=w_{y}+\frac{\mu_{x y}}{\mu_{x x}}\left(\frac{k_{y}}{k_{0}}-w_{x}\right)
$$

with its real and imaginary parts labelled as $\alpha=\alpha_{1}+\mathrm{i} \alpha_{2}$. The 'mass' and 'energy' in (21) are given by $m=(\beta-$ $\gamma) / 2$ and $\mathcal{E}=(\beta+\gamma) / 2$, where

$$
\beta=-\frac{\operatorname{det}\left[\boldsymbol{\mu}_{\|}\right]}{\mu_{x x}}, \gamma=-\epsilon_{z z}+\frac{\left|\frac{k_{y}}{k_{0}}-w_{x}\right|^{2}}{\mu_{x x}} .
$$

A similar formula to the above Dirac-like equation (21) holds for the $H_{z}$ polarization with permittivity and permeability interchanged, and $\boldsymbol{w} \rightarrow \boldsymbol{v}$. It should be emphasized that the meaning of 'energy' and 'mass' are here given in terms of material parameters through equation (23), which is conceptually similar to the treatment given in [17]. Despite this difference in interpretation from the true Dirac equation, we can apply the same index theorem summarized in (9-11) to identify the existence of 'topological' modes within electromagnetic materials, exactly as we did for the true Dirac equation in Sec. A.

a. Isotropic media: For the simplest case of a vanishing propagation constant, $k_{y}=0$, and isotropic materials $(\boldsymbol{\mu}=\mu \mathbf{1}, \boldsymbol{\epsilon}=\epsilon \mathbf{1}$ and $\boldsymbol{\xi}=\mathbf{0})$, our Dirac-like equation reproduces the recent findings of Shen et al. [34], where it was noticed that planar isotropic media could be understood in terms of a two component Dirac equation. We give a different viewpoint here, emphasizing the indifference of a bound mode to the details of the inhomogeneity. For these parameters our general Dirac equation (21) reduces to a simple form where $\alpha=0$ and the mass and energy are given in terms of the difference and the average of the permittivity and permeability, as we anticipated in the general case of equation (2)

$$
\begin{aligned}
m & =-\frac{1}{2}(\mu-\epsilon) \\
\mathcal{E} & =-\frac{1}{2}(\mu+\epsilon) .
\end{aligned}
$$


(a)

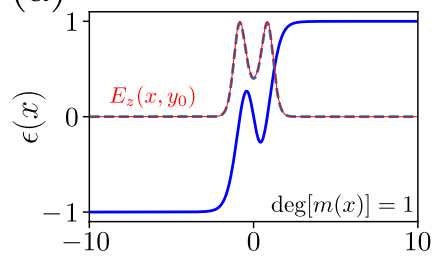

(b)
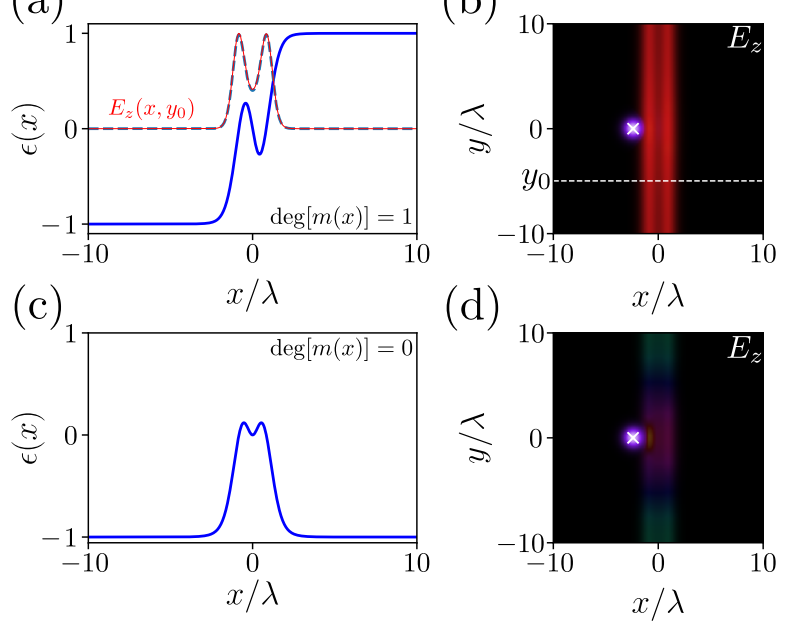

FIG. 3. A numerical illustration of the Jackiw-Rebbi mode in an isotropic medium where $\epsilon=-\mu=m(x)$. The blue curves in panels (a) and (c) show two different arbitrarily chosen permittivity profiles with degree +1 and 0 respectively. According to Eq. (10) a confined mode should exist for case (a) but not (c). Panels (b) and (d) show numerical simulations (COMSOL Multiphysics [33]; note periodic boundary conditions applied along $y$ ) for cases (a) and (c) respectively, where a line current source of frequency $k_{0}=2 \pi / \lambda$ is positioned at the white cross. In these plots the color indicates phase (red, green, cyan, purple) $\rightarrow(0, \pi / 2, \pi, 3 \pi / 2)$, and saturation indicates magnitude (scale arbitrary, but identical in (c) and (d)). The electric field $E_{z}$ along the line $y=y_{0}$ is plotted as the red line in panel (a) on top of which (blue dashed line) is plotted the analytical solution (9).

If the magnetic and electric responses vary in space, but are such that $\epsilon(x)=-\mu(x)=m(x)$ then the solutions to Maxwell's equations become equivalent to the zero energy modes of the Dirac equation (6), with a position dependent mass $m=m(x)$. In electromagnetic terms such a medium would not be expected to support any bound modes because the refractive index $n=\sqrt{\epsilon \mu}=\mathrm{i} m(x)$ is purely imaginary everywhere. This agrees with the Dirac picture sketched in the lower panel of Fig.1, where zero energy lies in the centre of the energy gap, and no propagation is possible. However, as we established in section A (see Eq. (8-11)) there can be modes in such a system. Their number is again governed by the degree of the function $m(x)$ (see Eq. (10)). Therefore an inhomogeneous medium where the permittivity and permeability have equal magnitude and opposite sign supports a single $k_{y}=0$ mode if the permittivity and permeability have different signs at $+\infty$ and $-\infty$. The details of the interface are irrelevant. This is demonstrated numerically in Fig. 3. One obvious concern with all the modes we find here is that they require a particular relationship between the material parameters. In appendix A we investigate the robustness of the mode shown in Fig. 3 to deviations from the condition $\epsilon=-\mu$.

It is interesting to compare this interface mode with the surface plasmon. In the electrostatic limit, the surface plasmon requires $\epsilon=-1=-\mu$, which is identical to the above condition. However, this limiting case of the plasmon is not the same mode we have found here. Firstly the plasmon exists at an interface with air, where $\epsilon=\mu=1$ (rather than $\epsilon=1=-\mu$ ), and secondly the electrostatic limit requires $k_{y} / k_{0} \rightarrow \infty$ rather than $k_{y}=0$, as we have for this mode. The mode given here requires the impedance $\sqrt{\mu / \epsilon}$ of the two media to be imaginary, but with each having a different sign, like the media investigated in [35-37].
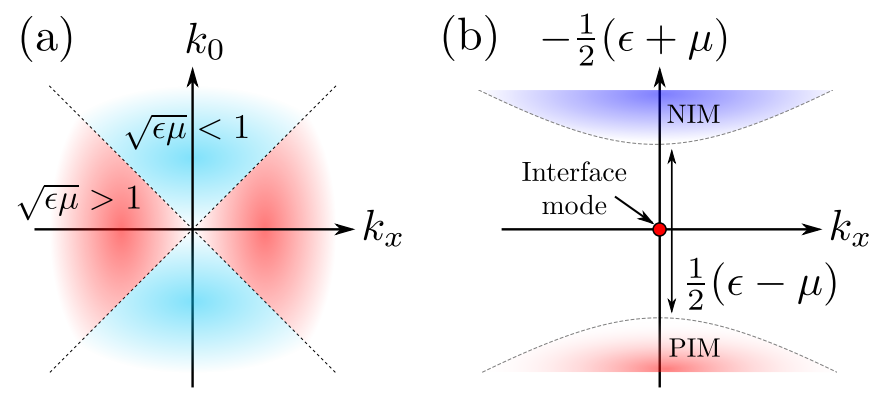

FIG. 4. Maxwell's equations in an isotropic material can be understood as a special case of the Dirac equation. (a) The effect of a material is usually understood in terms of the refractive index $n=\sqrt{\epsilon \mu}$, via the dispersion relation $k_{x}^{2}=n^{2} k_{0}^{2}$. A refractive index greater than unity moves the dispersion cone into the red region, and one of less than unity moves the dispersion into the blue region. (b) An alternative interpretation is to write the dispersion as $\left(k_{x} / k_{0}\right)^{2}=\mathcal{E}^{2}-m^{2}$, as given in Eq. (25). This shows that the region of disallowed propagation $|\epsilon+\mu|<|\epsilon-\mu|$ is equivalent to the 'mass gap' shown in the lower panel of Fig. 1, in this case separating regions of positive (PIM) and negative (NIM) index media. Accordingly there is a Jackiw-Rebbi like mode at $\mathcal{E}=-(\mu+\epsilon) / 2=0$, the existence of which depends only on the zero energy condition and the properties of $\mu$ and $\epsilon$ at infinity.

This analogy with the Dirac equation also reveals a rather unusual but informative way to understand the dispersion of electromagnetic waves in isotropic materials, which is worth commenting on. For the case of propagation along $x$ through a homogeneous medium the dispersion relation derived from (21) is given by

$$
\begin{aligned}
\left(\frac{k_{x}}{k_{0}}\right)^{2}=\mathcal{E}^{2}-m^{2} & =\left(\frac{\epsilon+\mu}{2}\right)^{2}-\left(\frac{\epsilon-\mu}{2}\right)^{2} \\
& =\epsilon \mu .
\end{aligned}
$$

This way of writing the equation reveals an interpretation of propagation in isotropic media in terms of an 'energy' and a 'mass', with propagation only possible when the sum of permittivity and permeability is greater in magnitude than their difference. This modified interpretation is sketched in Fig. 4. If we imagine a family of materials with a fixed difference between $\epsilon$ and $\mu$, this is analogous to a fixed mass in the Dirac equation, and results in a gap in the allowed values of the average $(\epsilon+\mu) / 2$, analogous to the 'mass gap' shown in the lower panel 
of Fig. 1. As an illustrative example consider a nonmagnetic material, $\mathcal{E}=-(1+\epsilon) / 2$ and $m=-(1-\epsilon) / 2$. The boundary between allowed and forbidden propagation is when $\mathcal{E}=m$, which in this case is when $\epsilon=0$, i.e. the tipping point between dielectric and metallic behaviour. The gap in the dispersion relation also closes when $m=0$, which equivalently is when $\epsilon=\mu$. This is the condition for impedance matching, and is when the effect of the material is equivalent to that of a coordinate transformation [8].

As a further comment, note that from (25) propagating waves are only possible when $\epsilon$ and $\mu$ have the same sign; when both are positive we have positive index, or 'right-handed' media, and when both are negative we have 'left-handed' or negative index media [38, 39]. The two signs of $\mathcal{E}$ in our analogy thus correspond respectively to positive and negative index media, as indicated by the colouring of the two regions of allowed propagation in Fig. 4b.

(a)
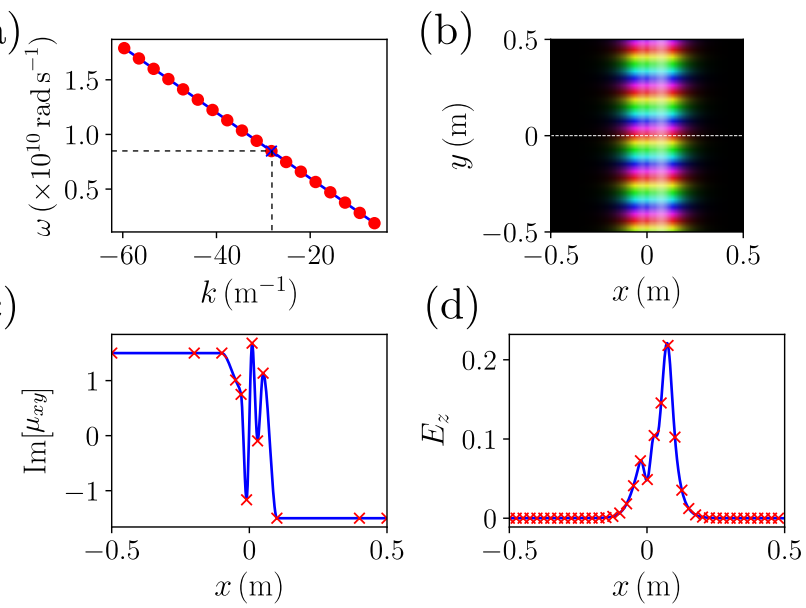

FIG. 5. One of the confined modes in a medium where the gyrotropy changes sign can be understood as Jackiw-Rebbi mode, and has a dispersion relation that is insensitive to how the gyrotropy parameter $\operatorname{Im}\left[\mu_{x y}\right]$ changes sign. Panel (a) compares the numerically calculated dispersion relation for one such mode (red dots) to the analytical prediction (blue solid line). The dashed black lines indicate the frequency and wave-vector of the numerically determined $E_{z}$ polarized mode shown in panel (b) (colouring as in Fig. 3). Panel (c) shows the spatial profile of the gyrotropy parameter, the central region of which was generated using an interpolation of a set of random numbers (red crosses). Panel (d) compares the numerical mode shape (blue solid line), evaluated along the white dashed line in panel (b), to the analytical prediction (9). In all these plots we have taken $\mu_{x x}=\epsilon_{z z}=1$

b. Gyrotropic media: A second example of these electromagnetic Jackiw-Rebbi modes is the case of a general complex Hermitian permeability $\boldsymbol{\mu}_{\|}$(zero bianisotropy $\boldsymbol{\xi}=\mathbf{0}$ ). A complex Hermitian form for either the permeability or the permittivity encodes the physical phenomenon known as gyrotropy [40], that has already been found to lead to unidirectional propagation of electromagnetic modes [14], modes that can be counted using topological invariants [15, 17, 32]. As found in [32], this is intimately connected with the properties of the Dirac equation. We now show that, without having to compute anything as complicated as a Chern number, very general statements can be made about such materials on the basis of the index theorem summarized in (9-11).

For these gyrotropic media we find that (21) reduces to

$$
\left(\begin{array}{cc}
0 & \mathcal{D} \\
\mathcal{D}^{\dagger} & 0
\end{array}\right)\left(\begin{array}{c}
E_{z} \\
\eta_{0} H_{y}
\end{array}\right)=\left(\begin{array}{cc}
\mathrm{i} \gamma & 0 \\
0 & -\mathrm{i} \beta
\end{array}\right)\left(\begin{array}{c}
E_{z} \\
\eta_{0} H_{y}
\end{array}\right)
$$

where $\mathcal{D}=k_{0}^{-1} \partial_{x}+\mathrm{i} \alpha_{1}-\alpha_{2}$. There are solutions to (26) that are in the kernel of either $\mathcal{D}$ or $\mathcal{D}^{\dagger}$

$$
\begin{aligned}
\mathcal{D}^{\dagger} E_{z} & =0 & \gamma & =\frac{1}{\mu_{x x}}\left(\frac{k_{y}}{k_{0}}\right)^{2}-\epsilon_{z z}=0, H_{y}=0 \\
\mathcal{D} H_{y} & =0 & \beta & =-\frac{\operatorname{det}\left[\boldsymbol{\mu}_{\|}\right]}{\mu_{x x}}=0, E_{z}=0 .
\end{aligned}
$$

The modes where $\mathcal{D}^{\dagger} E_{z}=0$ and $\mathcal{D} H_{y}=0$ are again of the form (9) and are respectively given by

$$
\begin{aligned}
& E_{z}=E_{0} \exp \left(-\frac{k_{y}}{\mu_{x x}} \int_{0}^{x}\left(\operatorname{Im}\left[\mu_{x y}\right]+\operatorname{iRe}\left[\mu_{x y}\right]\right) d x^{\prime}\right) \\
& H_{y}=H_{0} \exp \left(\frac{k_{y}}{\mu_{x x}} \int_{0}^{x}\left(\operatorname{Im}\left[\mu_{x y}\right]-\operatorname{iRe}\left[\mu_{x y}\right]\right) d x^{\prime}\right) .
\end{aligned}
$$

Whether these states can be normalized is determined by the sign of the imaginary part of $\mu_{y x}$. A comparison with (9-10) shows that the index of $\mathcal{D}$ is given by

$$
\operatorname{index}[\mathcal{D}]=-\operatorname{deg}\left[\frac{k_{y}}{\mu_{x x}} \operatorname{Im}\left[\mu_{x y}\right]\right]
$$

From Eq. (27) we can see that when the index equals -1, the propagation constant satisfies the dispersion relation $k_{y}^{2}=\epsilon_{z z} \mu_{x x} k_{0}^{2}$ (for simplicity it is assumed that $\epsilon_{z z} \mu_{x x}$ is constant in space). Eq. (29) also shows that this mode corresponds to a degree of $k_{y} \operatorname{Im}\left[\mu_{x y}\right] / \mu_{x x}$ equal to +1 . For $k_{y} / \mu_{x x}<0$, the imaginary part of $\mu_{x y}$ must thus be positive at $-\infty$ and negative at $+\infty$. This behaviour is verified in Fig. 5, where it is shown that the asymptotic behaviour of $\mu_{x y}$ (rather than the local details of the material) determines both the dispersion and the propagation direction of this electromagnetic mode. Note that this linearly dispersing interface mode is present irrespective of whether propagation is allowed in the bulk of the two media, meaning that the wave-vector along the interface is always greater than the wave-vector of propagating waves in the bulk of the two media (something already established in [17]). Note also that, as established in [17], the gyrotropy $\operatorname{Im}\left[\mu_{x y}\right]$ plays the role of the mass in the analogous Jackiw-Rebbi mode (9).

Eq. (27) shows that in the second case where $\operatorname{index}[\mathcal{D}]=1$ we must have $\beta=\operatorname{det}\left[\boldsymbol{\mu}_{\|}\right]=0$. This corresponds to the case where one of the eigenvalues of the permeability vanishes. As shown in Ref. [32], this is rather 
special point which can be understood as a zero in the refractive index for propagation in a complex direction. The mode corresponding to $\mathcal{D} H_{y}=0$ is unusual because it has no constraint on the magnitude of $k_{y}$ (i.e. the condition $\beta=0$ does not give rise to a dispersion relation, unlike $\gamma=0$ ). This 'unconstrained' part of the electromagnetic field was also found in [32], and has its value determined by the boundary conditions of the system. For example, if a magnetic mirror is placed anywhere along the $y$-axis this forces $H_{y}=0$, which eliminates this part of the field.
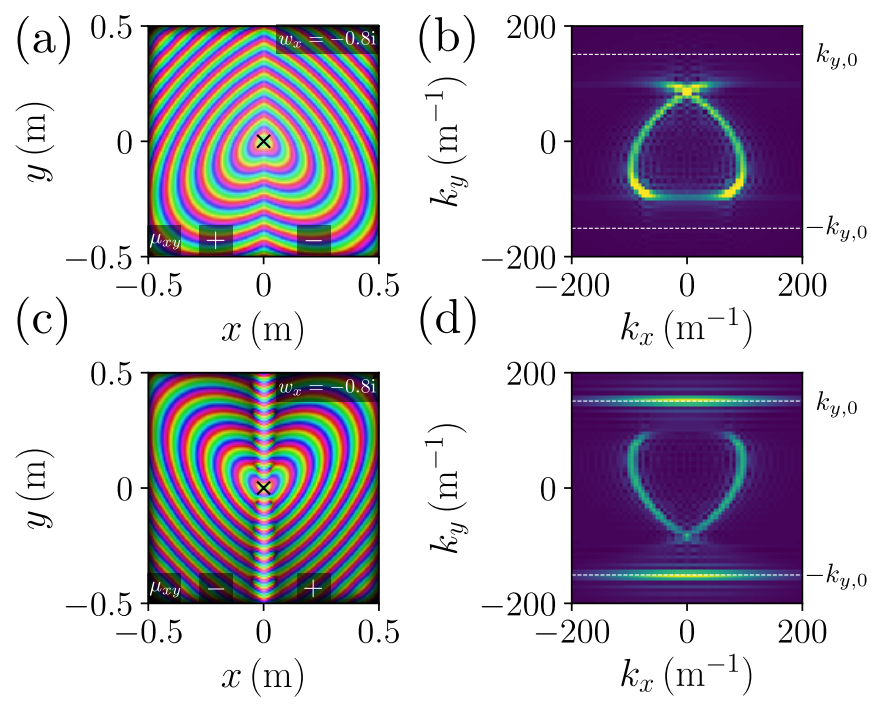

FIG. 6. Jackiw-Rebbi modes in anisotropic chiral media. The existence of these modes is governed by the sign of the product of the chirality and the permeability at infinity. Panel (a) shows the numerical simulation of the $E_{z}$ field, from an outof-plane line source (black cross), where the index of $\mathcal{D}$ is +1 , and we do not predict the presence of a localized mode. Here we made the arbitrary choice for the spatial profile of $\mu_{x y}=\mp \tanh (4 x / \lambda) / 2$, the asymptotic sign of which is shown at the bottom of each plot. In panel (c) we performed the same simulation for an index of $\mathcal{D}$ of -1 . The fixed chirality $\kappa_{x}=-\mathrm{i} w_{x}$ is indicated in the upper right corners of panels (a) and (c). The colours of the plots in panels (a) and (c) are as in Fig. 3. Panels (b) and (d) verify the predictions of Eq. (32) where we plot the numerical Fourier transform [41] of the fields in panels (a) and (c). The horizontal dashed white lines in panels (b) and (d) show the expected dispersion of the confined modes, $k_{y, 0}=k_{0} \sqrt{\mu_{x x} \epsilon_{z z}-\kappa_{x}^{2}}$. Note the parameters: $\epsilon_{z z}=\mu_{x x}=1, \mu_{x y} \in[-0.5,0.5], w_{y}=-\mathrm{i} \kappa_{y}=0$, and excitation frequency $f=c / \lambda=1.199 \times 10^{10} \mathrm{GHz}$.

c. Anisotropic chiral media: As a third example the medium exhibits a combination of anisotropy $\left(\mu_{x y}\right.$ real and non-zero) and chirality (the bianisotropy is imaginary $\boldsymbol{w}=\mathrm{i} \boldsymbol{\kappa})$. This case doesn't seem to have been considered before, and the optical Dirac equation (21) reduces to the equation (26) as in section $\mathrm{Cb}$. In this case the equivalent to the modes (28) are given by

$$
E_{z}=\exp \left\{k_{0} \int_{0}^{x}\left[\frac{\mu_{x y}}{\mu_{x x}}\left(\kappa_{x}-\frac{\mathrm{i} k_{y}}{k_{0}}\right)-\kappa_{y}\right] d x^{\prime}\right\}
$$

and

$$
H_{y}=\exp \left\{-k_{0} \int_{0}^{x}\left[\frac{\mu_{x y}}{\mu_{x x}}\left(\kappa_{x}-\mathrm{i} \frac{k_{y}}{k_{0}}\right)-\kappa_{y}\right] d x^{\prime}\right\} .
$$

Therefore the difference in the number of solutions to $\mathcal{D} H_{y}=0$ and $\mathcal{D}^{\dagger} E_{z}=0, N-\bar{N}$ is given by

$$
\operatorname{index}[\mathcal{D}]=\operatorname{deg}\left[\frac{\mu_{x y}}{\mu_{x x}} \kappa_{x}-\kappa_{y}\right] \text {. }
$$

We assume only the permeability component $\mu_{x y}$ changes with position, with everything else constant over space. If the degree equals -1 then, as in the previous example the mode must have the dispersion relation $k_{y}^{2}=$ $k_{0}^{2}\left(\mu_{x x} \epsilon_{z z}-\kappa_{x}^{2}\right)$, although in this case the sign of $k_{y}$ is not restricted. Fig. 6 shows a numerical verification of this effect, again for an arbitrary choice of spatially dependent $\mu_{x y}$. Note that, apart from the interface mode we have predicted, we have an unusual radiation pattern from the line source in Fig. 6, that is not symmetric in $y$. On either side of the interface, the materials are homogeneous and anisotropic, and we expect elliptical phase fronts. The radiation pattern of Fig. 6 can thus be understood as two sets of tilted elliptical phase fronts, respectively inverted around $x=0$, where they are joined.

In this example we have thus shown that our formalism predicts slightly unusual materials where a pair of linearly dispersing electromagnetic modes exist, provided only that $\kappa_{y}-\mu_{x y} \kappa_{x} / \mu_{x x}$ increases from a negative value at $-\infty$ to a positive one at $+\infty$, but not the reverse.

\section{Non-Hermitian materials}

So far our results concern bound modes within lossless media. In section we shall consider non-Hermitian media where the topologically determined modes are propagating rather than bound, showing that the topological invariant (11) can also govern the behaviour of propagating electromagnetic waves. Non-Hermitian systems have recently attracted interest in electromagnetism [42], providing a different route to realise reflectionless [43] and invisible [44] materials. There are some interesting implications of the above results in non-Hermitian systems. Analogues of the Jackiw-Rebbi modes exist in media with profiles of loss and gain. We shall show that the degree (11) controls not the index of the operator, but the character of the modes; either indicating a lack of reflection, coherent perfect absorption or lasing. This explains some recent findings of Makris and coworkers [45] who discovered a family of complex profiles that do not exhibit reflection, for an arbitrary amount of disorder. 
Although we could begin the discussion from our earlier general point of view (17), we can make the same point in a simple example. Take a fixed frequency, TE polarized wave propagating through an isotropic dielectric $(\mu=1)$ where the complex dielectric constant $\epsilon(x)$ varies in one spatial direction only. For propagation along $x$, Maxwell's equations reduce to the one dimensional Helmholtz equation for the wave amplitude $E_{z}$

$$
\left[\frac{d^{2}}{d x^{2}}+k_{0}^{2} \epsilon(x)\right] E_{z}=0
$$

Rather than considering separate field components as we did in the previous section, we now separate the electric field amplitude into its real and imaginary parts $E_{z}=$ $E_{1}+\mathrm{i} E_{2}$. This scalar equation for the complex variable $E_{z}$ thus becomes a pair of coupled equations for the real variables $E_{1}$ and $E_{2}$

$$
\left[\frac{d^{2}}{d x^{2}}+k_{0}^{2} \epsilon_{1}(x)-\mathrm{i} k_{0}^{2} \epsilon_{2}(x) \sigma_{y}\right]|\psi\rangle=0
$$

where $|\psi\rangle=\left(E_{1}, E_{2}\right)^{\mathrm{T}}$ and the permittivity was written $\epsilon=\epsilon_{1}+\mathrm{i} \epsilon_{2}$. For a certain class of complex permittivity profiles $\epsilon(x)$ the second order operator (34) can be written as the square of a Dirac operator

$$
\begin{aligned}
\mathcal{D}^{2} & =\left(-\mathrm{i} \sigma_{x} \frac{d}{d x}-\mathrm{i} \sigma_{z} k_{0} \alpha(x)\right)^{2} \\
& =-\frac{d^{2}}{d x^{2}}-k_{0}^{2} \alpha^{2}(x)+\mathrm{i} k_{0} \sigma_{y} \alpha^{\prime}(x)
\end{aligned}
$$

where $\alpha(x)$ is some real valued function of position. This idea is similar to that used by Longhi [46], who identified the transfer matrix formula as an effective Dirac operator. Equation (35) shows that when the Helmholtz operator equals $\mathcal{D}^{2}$, the permittivity is a complex function of the form

$$
\epsilon(x)=\alpha^{2}(x)+\frac{\mathrm{i}}{k_{0}} \alpha^{\prime}(x) .
$$

This form of the permittivity was recently considered in [45] because they found that, however disordered such a profile, it could support waves that propagate without either backscattering or intensity variation. Here we see these properties are a consequence of the factorization given in (35), and are another example of Jackiw-Rebbi modes appearing in electromagnetism.

One of the solutions to equation (33) is that where $\mathcal{D}|\psi\rangle=0$, which is again the one dimensional Dirac equation for zero energy

$$
\left[-\mathrm{i} \sigma_{x} \frac{d}{d x}-\mathrm{i} \sigma_{z} k_{0} \alpha(x)\right]|\psi\rangle=0 .
$$

As we have seen many times, there are solutions (9) to this equation not governed by the detailed behaviour of the 'mass' $m(x)$, but by its value at $\pm \infty$. The difference in this case is that the 'mass' is an imaginary quantity
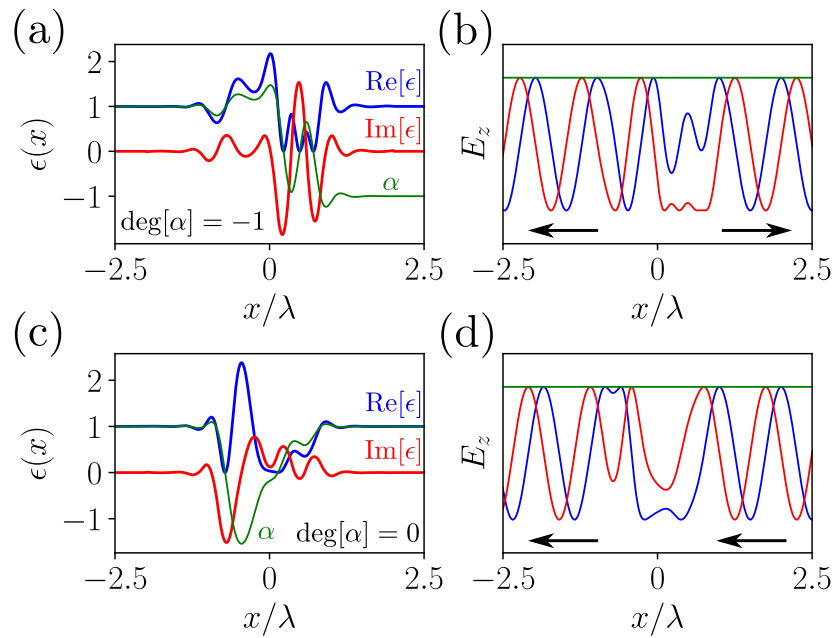

FIG. 7. For the complex permittivity profiles (36), the Helmholtz equation becomes an effective Dirac equation with an imaginary mass $m=-\mathrm{i} k_{0} \alpha(x)$. Two example profiles (central region constructed using an interpolation of random numbers) are given in panels (a) and (c), with the corresponding waves in panels (b) and (d) (real part blue, imaginary part red, and magnitude green). The wave equation was integrated numerically using functions from the PYTHON Scipy library [41]. For imaginary mass, the Jackiw-Rebbi modes (9) are constant amplitude travelling waves, and the degree of $\alpha$ determines, via (40), whether the mode is a travelling wave, is purely out-going, or purely in-coming.

$m(x)=-\mathrm{i} k_{0} \alpha(x)$ and the two solutions (9) to Eq. (37) are given by

$$
|\psi\rangle=\frac{1}{2}\left\{\begin{array}{l}
a_{y,+} \mathrm{e}^{\mathrm{i} \int_{0}^{x} \alpha\left(x^{\prime}\right) d x^{\prime}}+a_{y,-} \mathrm{e}^{-\mathrm{i} \int_{0}^{x} \alpha\left(x^{\prime}\right) d x^{\prime}} \\
\mathrm{i} a_{y,-} \mathrm{e}^{-\mathrm{i} \int_{0}^{x} \alpha\left(x^{\prime}\right) d x^{\prime}}-\mathrm{i} a_{y,+} \mathrm{e}^{\mathrm{i} \int_{0}^{x} \alpha\left(x^{\prime}\right) d x^{\prime}}
\end{array}\right.
$$

where the linear combinations of (9) have been chosen so that the components of $|\psi\rangle$ are real valued. In the case of an imaginary mass, the degree of $\operatorname{Im}[m(x)]$ is not related to the kernel of $\mathcal{D}$. This is because the 'mass' now only controls the phase of the solution, and whatever its sign at infinity this is irrelevant to the norm of $|\psi\rangle$. Nevertheless the degree of $\alpha$ still controls something important about the wave.

Writing the solution (38) in component form we have

$$
|\psi\rangle=\left(\begin{array}{c}
\cos \left(\int_{0}^{x} \alpha\left(x^{\prime}\right) d x^{\prime}\right) \\
-\sin \left(\int_{0}^{x} \alpha\left(x^{\prime}\right) d x^{\prime}\right)
\end{array}\right),\left(\begin{array}{c}
\sin \left(\int_{0}^{x} \alpha\left(x^{\prime}\right) d x^{\prime}\right) \\
\cos \left(\int_{0}^{x} \alpha\left(x^{\prime}\right) d x^{\prime}\right)
\end{array}\right)
$$

both of which correspond to the same wave, $E_{z}=$ $\exp \left(-\mathrm{i} \int_{0}^{x} \alpha\left(x^{\prime}\right) d x^{\prime}\right)$ which is left going if $\alpha$ is positive as $x \rightarrow \pm \infty$. We can thus see that if the degree of $\alpha$ is zero, the material supports a wave of constant amplitude that propagates either to the left or the right, depending on the sign of $\alpha$, without reflection. Meanwhile if the degree of $\alpha$ is -1 the wave is outgoing on both the right and the left hand side of the profile, and the material thus acts as a 'laser'. Finally, for a degree of +1 the wave is incoming 
on the left and the right of the profile and we have socalled coherent perfect absorption (CPA) [47, 48]. Lasing and coherent perfect absorption are the time reverse of one another, and are fundamentally non-Hermitian phenomena, where a pole or a zero of the scattering matrix is present at a real valued excitation frequency [47]. In these cases the material acts either as a perfect source, or a perfect drain for wave energy. The relationship between $\alpha(x)$ and the character of our modes can be summarized as follows

$$
\operatorname{deg}[\alpha(x)]= \begin{cases}+1 & (\mathrm{CPA}) \\ -1 & (\text { Lasing }) \\ 0 & \text { (No reflection) }\end{cases}
$$

Fig. 7 shows a numerical demonstration of this effect, where two $\alpha$ profiles have been constructed using an interpolation of random numbers, with $|\alpha| \rightarrow 1$ at infinity. Although no longer a consequence of the Atiyah-Singer index theorem, the degree of $\alpha(x)$ appearing in the permittivity profiles (36) determines something about the wave that is again independent of the detailed behaviour of the material profile.

\section{E. Summary and Conclusions}

In this work we investigated the electromagnetic analogues of the Jackiw-Rebbi modes of the Dirac equation, illustrating that for some families of stratified electromagnetic materials, one can vary the material in an arbitrary fashion without changing the dispersion of one of the bound modes. The examples considered here show that known modes of both isotropic and gyrotropic media can be understood in this way, and one can also predict new unusual modes such as the example given for anisotropic chiral materials. In all these cases the existence of the mode can be determined using the same simple topological invariant.

Finally we showed in section D, that these applications are not restricted to bound states and one can use the same topological invariant to predict the character of non-Hermitian media via formula (40). This result showed that the recent discovery of disordered scattering free non-Hermitian media [45] is, rather surprisingly, actually an instance of a Jackiw-Rebbi mode in electromagnetism.

As a final comment, we note that we found for stratified media that the Maxwell equations can be written as a four component Dirac equation, from which we can find the general solution as the path ordered product (19). Aside from the examples given here, there seem to be many more interesting applications of this formula.

\section{ACKNOWLEDGMENTS}

SARH acknowledges useful conversations with Bill Barnes, Tom Philbin, and a series of illuminating lec- tures from Andrey Shytov. SARH is funded by the Royal Society and TATA (RPG-2016-186). 


\section{Appendix A: Robustness of profiles}

One obvious question is whether the modes we predict are robust to deviations from the zero energy condition. In this electromagnetic case the zero energy condition is a restriction relating the spatial profile of different material parameters. For example, in the case of isotropic media, this was equivalent to the requirement that $\epsilon=-\mu$, which in practice may be difficult to achieve. There is of course a huge space of possible deviations from perfection. In this appendix we perform some initial investigations. In Fig. 8 we investigate the robustness of the mode shown in Fig. 3 of the main text. We look at deviations from the condition $\epsilon=-\mu$ through introducing a scale factor $s, \epsilon=-s \mu$. As shown in the three panels of Fig. 8, there is a surprising insensitivity to this condition, with almost the same mode evident when $\epsilon=-\mu / 2$. Figures 9 and 10 show the effect of deviations of the permittivity from being homogeneous, for the gyrotropic and anisotropic chiral media given in Figures 5-6 of the main text.
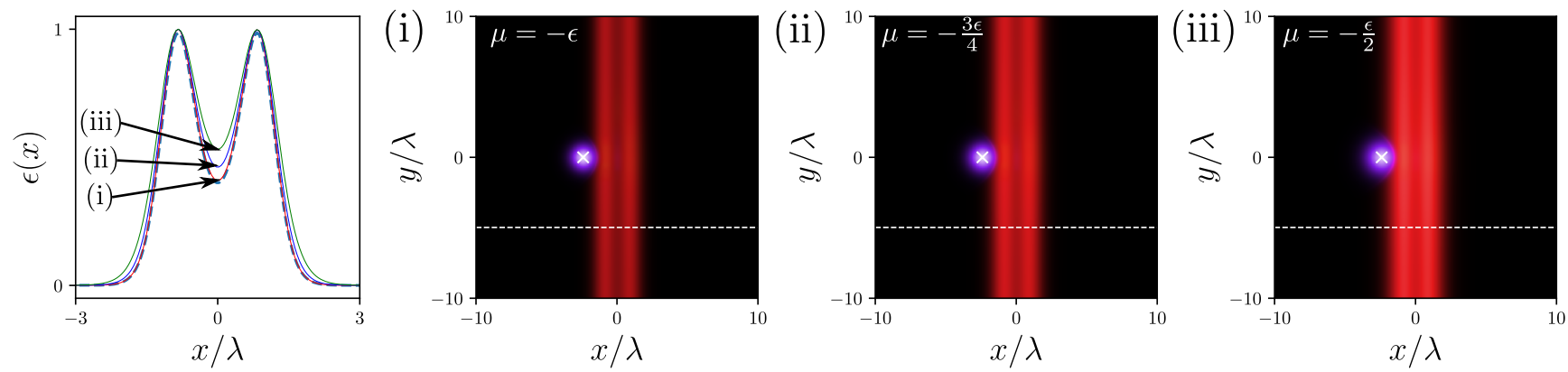

FIG. 8. The effect of deviations from the zero energy condition, for the isotropic material investigated in Fig. 3. All parameters identical to Fig. 3, except the profile $\mu(x)$ is now proportional to $-\epsilon(x)$, with the proportionality constants shown in panels (i-iii). The colour scheme is the same as that used in Fig. 4s, and the saturation scale is identical in panels (i-iii). The leftmost panel shows the normalized mode profiles evaluated along the white dashed lines in (i-iii), with the black dashed line showing the mode profile determined analytically from the zero energy condition.
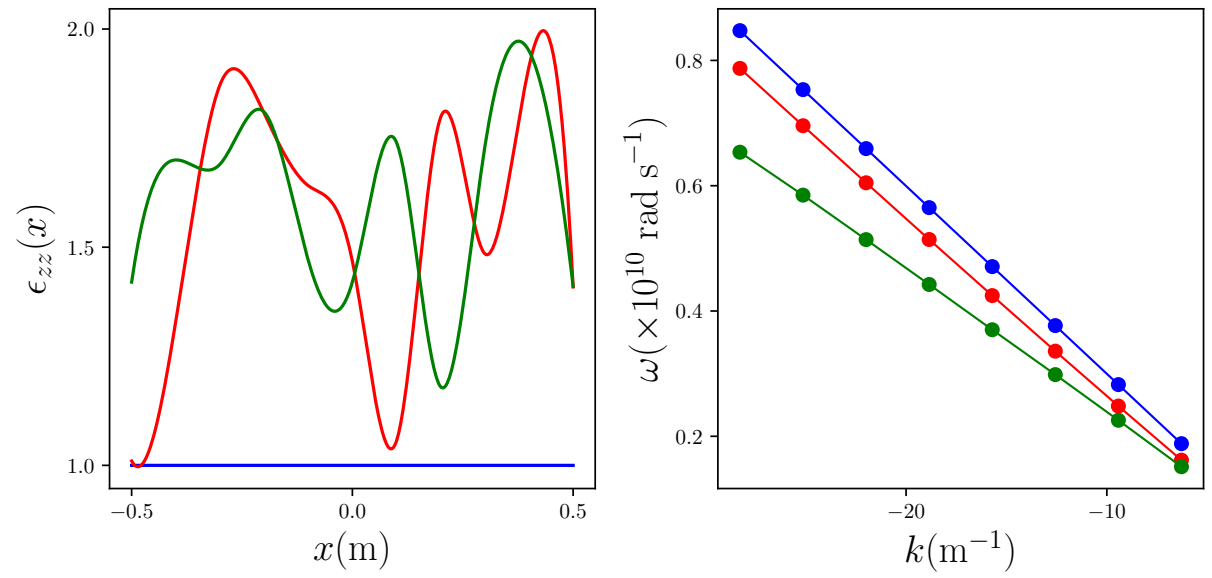

FIG. 9. In the main text we considered gyrotropic media supporting a Jackiw-Rebbi mode with a linear dispersion relation that was insensitive to the spatial variation of the gyrotropy (see Fig. 5). To obtain this mode we assumed a uniform out of plane permittivity. Here we investigate the effect of making the out of plane permittivity inhomogeneous. A spline fit through a set of random numbers was used to generate the two inhomogeneous permittivity profiles in panel (a) (uniform case also included, for reference). Panel (b) shows the corresponding dispersion relation of the lowest order mode (found using COMSOL multiphysics, as in Fig. 5) for the three cases shown in panel (a). As would be expected from the dispersion relation $k_{y}^{2}=\epsilon_{z z} \mu_{x x} k_{0}^{2}$, the slope of the dispersion changes (the value of the out of plane permittivity is now different where the mode is confined), however the mode remains unidirectional and the dispersion remains extemely close to linear. 
(a)

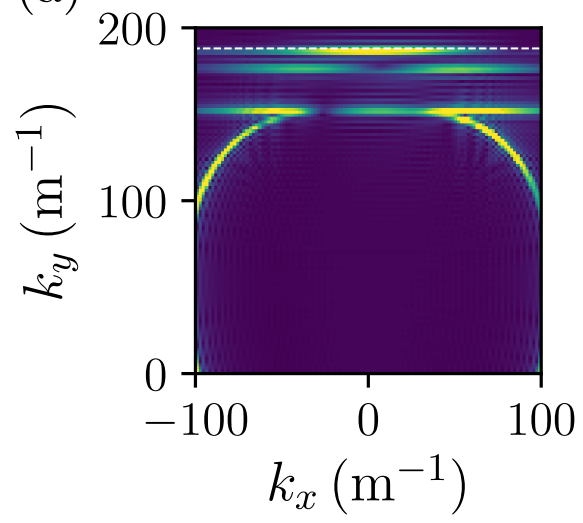

(b)

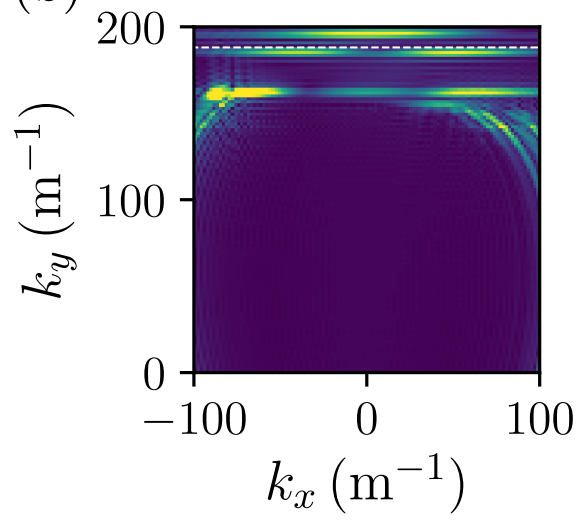

(c)

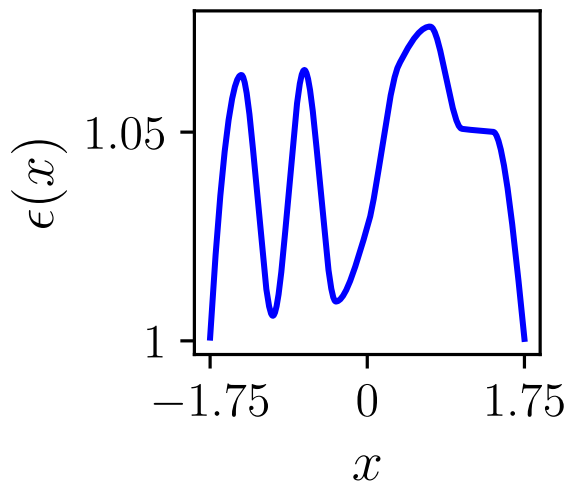

FIG. 10. As in Figs. 8 and 9, we show the effect of a spatially inhomogeneous permittivity on the modes of the anisotropic chiral medium shown in Fig. 6 (here some of the simulation parameters are changed from Fig. 6, with a 12 times bigger simulation area, a randomly generated profile for $\mu_{x y}$, and $\mu_{x x}=2$ ). In panel (a) the Fourier transform of the point source field for a uniform permittivity is shown, with the dashed line indicating the expected value of $k_{y}$ for the Jackiw-Rebbi mode. In panel (b), the permittivity is given by the spatial profile shown in panel (c), and the Jackiw-Rebbi mode is still clearly visible, but with an increased wave-vector, as expected from the increase in permittivity indicated in panel (c).

[1] M. Bordag, U. Mohideen, and M. Mostepanenko. New developments in the casimir effect. Phys. Rep., 353:1, 2001.

[2] N. Nagaosa and Y. Tokura. Topological properties and dynamics of magnetic Skyrmions. Nature Nanotech., 8:899, 2013.

[3] L. D. Landau and E. M. Lifshitz. Theory of Elasticity. Butterworth-Heinemann, 1999.

[4] M. Srednicki. Quantum Field Theory. Cambridge University Press, 2007.

[5] S. Carlip. Quantum Gravity in 2+1 Dimensions. Cambridge University Press, 2003.

[6] M. Z. Hasan and C. L. Kane. Colloquium: Topological insulators. Rev. Mod. Phys., 82:3045, 2010.

[7] A. Greenleaf, M. Lassas, and G. Uhlmann. On nonuniqueness for Calderon's inverse problem. Math. Res. Lett., 10:685, 2003.

[8] J. B. Pendry, D. Schurig, and D. R. Smith. Controlling Electromagnetic Fields. Science, 312:1780, 2006.

[9] U. Leonhardt. Optical Conformal Mapping. Science, 312:1777, 2006.

[10] F. D. M. Haldane and S. Raghu. Possible realization of directional optical waveguides in photonic crystals with broken time-reversal symmetry. Phys. Rev. Lett., 100:013904, 2008.

[11] Z. Wang, Y. Chong, and M. Joannopoulos, J. D. amd Soljačić. Observation of unidirectional backscattering-immune topological electromagnetic states. Nature, 461:772, 2009.

[12] A. B. Khanikaev, S. H. Mousavi, W.-K. Tse, M. Kargarian, A. H. MacDonald, and G. Shvets. Photonic topological insulators. Nature Mat., page 233, 2013.

[13] L. Lu, J. D. Joannopoulos, and M. Soljačić. Topological photonics. Nature Phot., 8:821, 2014.

[14] A. R. Davoyan and N. Engheta. Theory of Wave Propagation in Magnetized Near-Zero-Epsilon Metamaterials:
Evidence for One-Way Photonic States and Magnetically Switched Transparency and Opacity. Phys. Rev. Lett., 111:257401, 2013.

[15] M. G. Silveirinha. Bulk-edge correspondence for topological photonic continua. Phys. Rev. B, 94:205105, 2016.

[16] M. G. Silveirinha. Topological classification of Cherntype insulators by means of the photonic Green function. Phys. Rev. B, 97:115146, 2018.

[17] S. A. R. Horsley. Topology and the optical Dirac equation. Phys. Rev. A, 98:043837, 2018.

[18] T. Van Mechelen and Z. Jacob. Photonic Dirac monopoles and Skyrmions: spin-1 quantization. Opt. Mat. Exp., 9:95, 2019.

[19] S. M. Barnett. Optical dirac equation. New J. Phys., 16:093008, 2014.

[20] M. C. Rechtsman, J. M. Zeuner, Y. Plotnik, Y. Lumer, D. Podolsky, F. Dreisow, S. Nolte, M. Segev, and A. Szameit. Photonic floquet topological insulators. Nature, 496:196, 2013.

[21] J. Mei, Y. Wu, C. T. Chan, and Z.-Q. Zhang. Firstprinciples study of dirac and dirac-like cones in phononic and photonic crystals. Phys. Rev. B, 86:035141, 2012.

[22] M. F. Atiyah and I. M. Singer. The index of Elliptic Operators on Compact Manifolds. Bull. Amer. Math. Soc., 69:422, 1963.

[23] S. Rosenberg. The Laplacian on a Riemannian manifold. Cambridge University Press, 1997.

[24] G. E. Volovik. The Universe in a Helium Droplet. Clarendon Press, 2003.

[25] A. J. Neimi and G. W. Semenoff. Spectral asymmetry on an open space. Phys. Rev. D, 30:809, 1984.

[26] A. Wassermann. The Atiyah-Singer index theorem, Lent 2010 (lecture notes). https://www.dpmms.cam.ac.uk/ ajw/AS10.pdf. Accessed: 2019-01-23.

[27] E. Getzler. A short proof of the local Atiyah-Singer index theorem. Topology, 25:111, 1986. 
[28] A. Mostafazadeh. Supersymmetry, Path Integration, and the Atiyah-Singer Index Theorem. Dissertation, University of Texas (arXiv:hep-th/9405048), 1994.

[29] R. Jackiw and C. Rebbi. Solitons with fermion number . Phys. Rev. D, 13:3398, 1976.

[30] E. Outerelo and J. M. Ruiz. Mapping Degree Theory. American Mathematical Society, 2009.

[31] E. Witten. Supersymmetry and Morse theory. J. Diff. Geom., 17:661, 1982.

[32] Horsley. S. A. R. Unidirectional propagation and complex principal axes. Phys. Rev. A, 97:023834, 2018.

[33] COMSOL Multiphysics (R) v. http://www.comsol.com.

[34] W. Tan, Y. Sun, H. Chen, and S.-Q. Shen. Photonic simulation of topological excitations in metamaterials. Sci. Rep., (3842), 2014.

[35] Alú, A. and Engheta, N. Pairing an Epsilon-Negative Slab With a Mu-Negative Slab: Resonance, Tunneling and Transparency. IEEE Trans. Ant. Prop., 51:2558, 2003.

[36] S. A. R. Horsley. One dimensional electromagnetic waves on flat surfaces. J. Phys. D, 47:435103.

[37] D. J. Bisharat and D. F. Sievenpiper. Guiding Waves Along an Infinitesimal Line between Impedance Surfaces. Phys. Rev. Lett., 119:106802, 2017.

[38] V. G. Veselago. The Electrodynamics of Substances with Simultaneously Negative Values of $\epsilon$ and $\mu$. Sov. Phys. Usp., 10:509, 1968.
[39] J. B. Pendry. Negative refraction makes a perfect lens. Phys. Rev. Lett., 85:3966, 2000.

[40] L. D. Landau and E. M. Lifshitz. Electrodynamics of Continuous Media. Butterworth-Heinemann, 2004.

[41] Eric Jones, Travis Oliphant, Pearu Peterson, and et al. SciPy: Open source scientific tools for Python, 2001-. [Online; accessed 2019-03-18].

[42] S. Longhi. Parity-time symmetry meets photonics: A new twist in non-hermitian optics. Europhys. Lett., 120:64001, 2018.

[43] S. A. R. Horsley, M. Artoni, and G. C. La Rocca. Spatial Kramers-Kronig relations and the reflection of waves. Nat. Phot., 9:436, 2015.

[44] Z. Lin, H. Ramezani, T. Eichelkraut, T. Kottos, H. Cao, and D. N. Christodoulides. Unidirectional Invisibility Induced by $\mathcal{P} \mathcal{T}-$ Symmetric Periodic Structures. Phys. Rev. Lett., 106:213901, 2011.

[45] K. G. Makris, A. Brandstötter, P. Ambichl, Z. H. Musslimani, and S. Rotter. Wave propagation through disordered media without backscattering and intensity variations. Light: Science \& Applications, 6:e17035, 2017.

[46] S. Longhi. Optical Realization of Relativistic NonHermitian Quantum Mechanics. Phys. Rev. Lett., 105:013903, 2010.

[47] Y. D. Chong, L. Ge, H. Cao, and A. D. Stone. Coherent perfect absorbers: Time-reversed lasers. Phys. Rev. Lett., 105:053901, 2010.

[48] S. Longhi. $\mathcal{P} \mathcal{T}$-symmetric laser absorber. Phys. Rev. A, 82:031801, 2010. 